\title{
THz Absorber for Breast Cancer Early Detection based on Graphene as Multi-layer Structure
}

Mohammad Reza Soheilifar ( $\nabla$ rsoheilifar@ee.kntu.ac.ir)

Imam khomeyini university https://orcid.org/0000-0001-7739-7489

Afrooz Rezazadeh

departement of electrical engineering, allameh mohaddes nouri

\section{Research Article}

Keywords: Absorber, Terahertz, graphene, cancer detection, sensitivity

Posted Date: July 19th, 2021

DOI: https://doi.org/10.21203/rs.3.rs-698664/v1

License: (9) This work is licensed under a Creative Commons Attribution 4.0 International License. Read Full License 


\title{
THz absorber for breast cancer early detection based on graphene as multi-layer structure
}

\author{
${ }^{1}$ Afrooz Rezazadeh, ${ }^{2}$ Mohammad Reza Soheilifar \\ ${ }^{1}$ Department of Electrical Engineering, Allameh Mohaddes Nouri University. Iran \\ ${ }^{2}$ Faculty of Electrical Engineering of Naval sciences, Imam Khomeyni University of Nowshahr, Iran \\ Corresponding author: rsoheilifar@ee.kntu.ac.ir
}

\begin{abstract}
Today, the cost of treating cancer is very high, so early detection of cancer is essential, and one of the possible ways to diagnose cancer in the early stages is to use sensors that are made in the terahertz area. The proposed structure is a multilayer structure in which graphene is used in the upper and middle layer and the middle layer has a ribbon form. A disk form element is used in the upper layer. Finally, a ring is added to the structure. In the final structure of the ring, it actually inhibits the field, and this will increase the Q-factor at 6.7 THz. The interaction of layers on each other and their effect on reflection is studied to modify the final structure by variation of the chemical potential of the graphene layer in the range of 0.2 to $0.6 \mathrm{eV}$. Finally, the proposed absorber is used to discriminate cancer and healthy breast tissue. The effect of the thickness and distance of the tissue from the absorber are examined to realize the material effect on sensitivity and figure of merit (FOM) as two main factors for detecting the cancer tissue.
\end{abstract}

Index Terms -Absorber; Terahertz; graphene; cancer detection; sensitivity

\section{Introduction}

Early cancer detection plays an important role for the medical centers in the microwave [1], THz [2], and optical systems [3]. For this aim, various systems have been developed in the microwave spectrum for detecting breast cancer tumors during past decades [4] and microwave antenna is the main element for these systems [5]. However, by recent development in micro and Nanofabrication, the $\mathrm{THz}$ and optical devices get more consideration for early cancerdetecting [6]. In fact, a small sample is sufficient for the $\mathrm{THz}$ spectroscopy system based on $\mathrm{THz}$ domain spectroscopy (TDS) [7] to recognize the cancer tumor from the normal tissue by analyzing either the pulse response [8] or frequency shift [9].

In addition, the $\mathrm{THz}$ technology has been considered more for non-ionized characteristics [10] and therefore various devices have been designed such as antenna [11], filter [12], metasurface [13], and absorber [14]. Many models of the THz absorbers have been developed for various applications such as $\mathrm{THz}$ sensing [15]. For example, split-ring resonators [16] and Jerusalem cross [17] have been studied for $\mathrm{THz}$ application. The $\mathrm{THz}$ absorber is more interesting because the energy can be concentrated [18]. Thus the sensitivity and
Figure of merit (FOM) will increase which are vital for the sensors [19].

On the other hand, various techniques base on special material have been used to design the sensors [20], and using graphene is known as a conventional technique for developing $\mathrm{THz}$ absorber because of the plasmonic behavior of the graphene at this spectrum $[21,22]$. Recently, many models of absorber by graphene have been investigated for narrowband $[23,24]$ or UWB spectrum [25, 26].

The main purpose of this paper is to design an absorber for the terahertz spectrum, which uses graphene in the design of this absorber and presents a multi-layered structure that has a high Q-factor capability. Then we model two different types of skin and breast cancer. Finally, we place these cancerous tissues on the absorber structure and as a result, we obtain sensitivity and Figure of merit. So, three parameters of Qfactor, sensitivity, and FOM are determined for cancer and healthy tissue.

\section{The graphene absorber designing}

Graphene is a type of carbon allotropes. It is composed of a two-dimensional honeycomb crystal structure in which each carbon atom is attached to three other carbon atoms. The conductivity of graphene according to the Kubo formula has two parts, inter and intra, which are shown in the Eq.1 and 2, and the final conductivity of graphene can be obtained from the sum of these two parts [27].

$$
\begin{aligned}
& \sigma^{\operatorname{intra}}(\omega)=\frac{e^{2} K_{B} T}{\pi \mathrm{h}^{2}(\omega-i 2 \Gamma)}\left[\frac{\mu_{c}}{K_{B} T}+2 \ln \left(e^{-\frac{\mu_{c}}{K_{B} T}}+1\right)\right] \\
& \sigma^{\operatorname{inter}(\omega)}=\frac{e^{2}}{4 \pi \mathrm{h}} \ln \left(\frac{2\left|\mu_{c}\right|-(\omega-i 2 \Gamma) \mathrm{h}}{2\left|\mu_{c}\right|+(\omega-i 2 \Gamma) \mathrm{h}}\right)
\end{aligned}
$$

Here $\sigma$ is the surface conductivity and e electric charge, $\omega$ frequency angle, $K_{B}$ Planck constant, $T$ ambient temperature, KB Boltzmann constant, $\mu \mathrm{c}$ chemical potential, $\tau$ relaxation time and $\Gamma$ electron diffraction velocity and can be defined by $\tau=1 / 2 \Gamma$. 
The electrical conductivity of the graphene layer can be obtained by Eq.3, where $\sigma$ is the conductivity of the graphene layer, which is obtained from the Kubo equation of Eq.1 and 2 , and $\omega$ is the angular frequency and thickness $d$ of the graphene layer [28].

$\varepsilon_{r}(d)=1-j \frac{\sigma}{\omega \varepsilon_{0} d}$

The proposed absorber is a multi-layered structure that at the bottom layer of a gold plate is located as a reflector. Then a quartz substrate is placed on the gold and graphene strips are placed on the first substrate. Then another layer of quartz material is placed on the graphene layer and finally, a graphene element is placed on it as the main element.

The proposed absorber is a multi-layered structure and the two absorbers are designed to be used as sensors. In fact, the difference between the two structures is in the main absorber element. The proposed multi-layer structure has a gold layer in the bottom as a reflector with a thickness of 0.5 $\mu \mathrm{m}$, which is used to eliminate the transmission, and on it is placed the main substrate with a thickness of $4 \mu \mathrm{m}$. The graphene ribbons with a width of $3 \mu \mathrm{m}$ are placed on the first substrate and the distance between these ribbons is assumed to be $3 \mu \mathrm{m}$, and this layer is presented in Fig.1 (a). Then we put a dielectric layer on the graphene ribbons and the thickness of this layer is assumed to be $3 \mu \mathrm{m}$ and all the dielectrics are made of quartz with a permittivity of 3.75 . Finally, a graphene element is placed on the second substrate. The boundary condition is one of the most important parts of an absorber simulation. Here, we have assumed perfect electrical conductor (PEC) in one direction and perfect magnetic conductor (PMC) in the other direction to provide periodic boundary conditions. For exciting the absorber, waveguide port excitation and periodic boundary conditions are used.

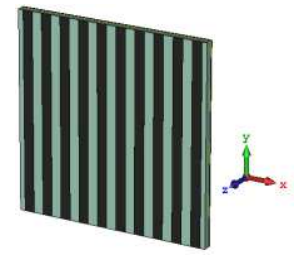

(a)

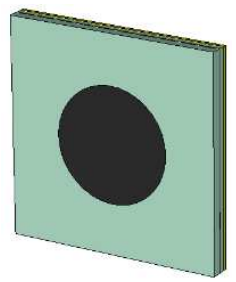

(b)

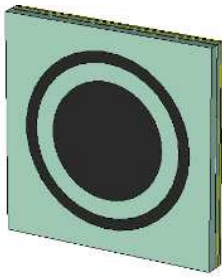

(c)
Fig.1 Proposed structure (a) Graphene ribbons on first substrate (b) Graphene disk (c) Graphene disk and ring in the final structure

\section{The simulation results and discussion}

As shown in Fig.2, the first structure has a resonance at the frequency of $6.7 \mathrm{THz}$. However, in the final structure, a disk and a loop have been used to improve the reflection, and the final structure has a resonance at same frequency with less reflection and is presented in Fig.2. As can be seen, by using the ring structure, the amount of reflection is drastically reduced from $-15 \mathrm{~dB}$ to $-20 \mathrm{~dB}$, which means having a stronger absorber as well as improving the Q-factor, which can be achieved through Eq.4 [29] for the primary absorber with disk element the Q-factor is obtained 419.4 and for the proposed absorber when the ring added the Q-factor increases to 670 .

$Q=\frac{f_{0}}{B W_{3 d B}}$

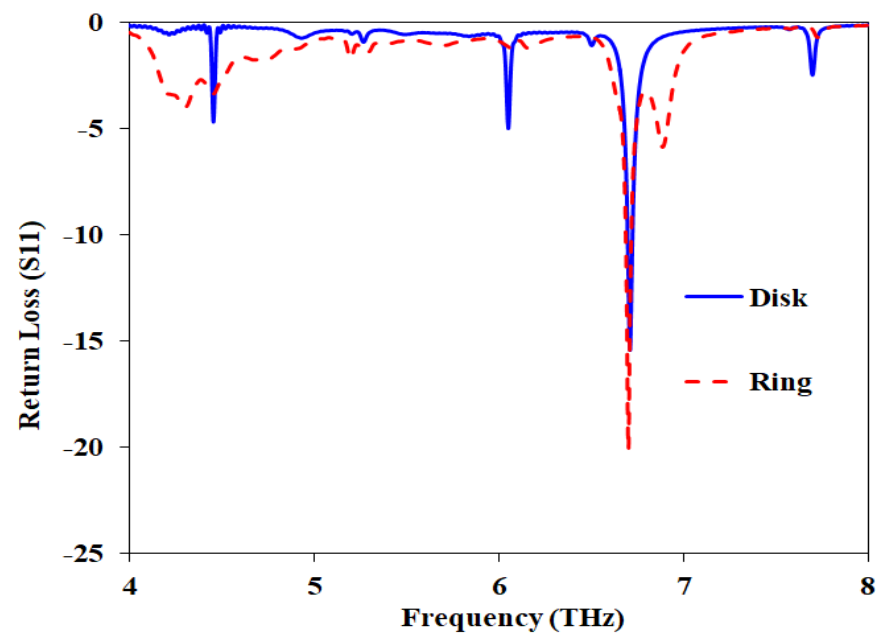

Fig.2 the reflection of proposed absorbers

Two factors, chemical potential and relaxation time will affect the frequency response of our structure. First, we consider the chemical potential of the ribbons as $0.2 \mathrm{eV}$ and the changes in the chemical potential of the main element (the disk and the disk and ring) for the chemical potentials of 0.2 , 0.4, and $0.6 \mathrm{eV}$. As shown in Fig. 3 (a) for a chemical potential of $0.2 \mathrm{eV}$ of the disk for the primary absorber, the structure has two resonances, but the reflection is not very significant and the reflection is about -9 to $-11 \mathrm{~dB}$. Then, with increasing chemical potential to 0.4 and $0.6 \mathrm{eV}$, the primary absorber has one resonance, but it has a lower reflection which reaches about -15 to $-17 \mathrm{~dB}$. Fig.3 (b) shows that the final structure has dual-band properties for a chemical potential of $0.2 \mathrm{eV}$ for the disk and ring with a chemical potential of the $0.2 \mathrm{eV}$ for ribbons, but both resonances are in the frequency range of 7 to $8 \mathrm{THz}$, but by increasing chemical potential, the structure has a resonance at lower frequencies in it happens about 6.7 THz. 


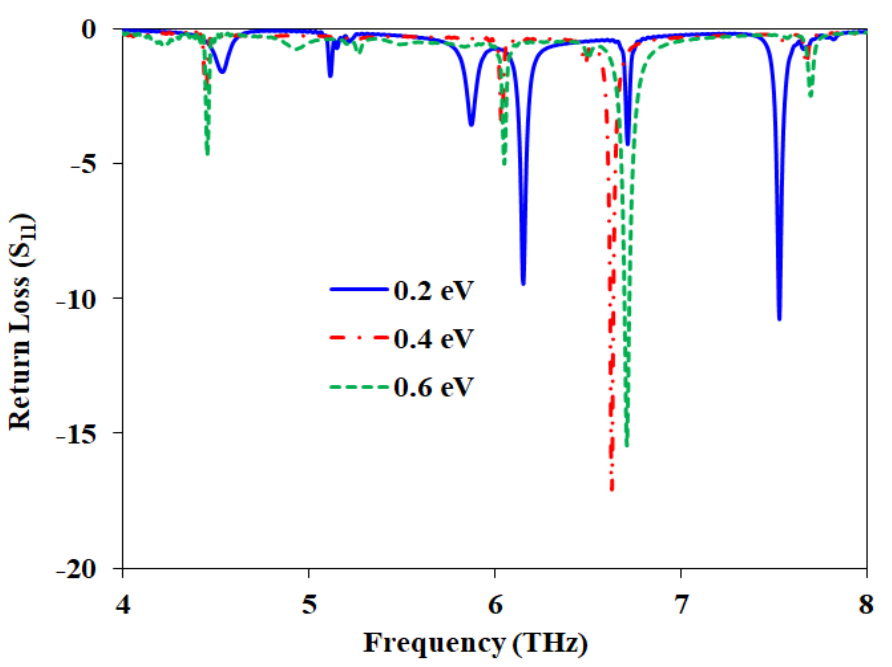

(a)

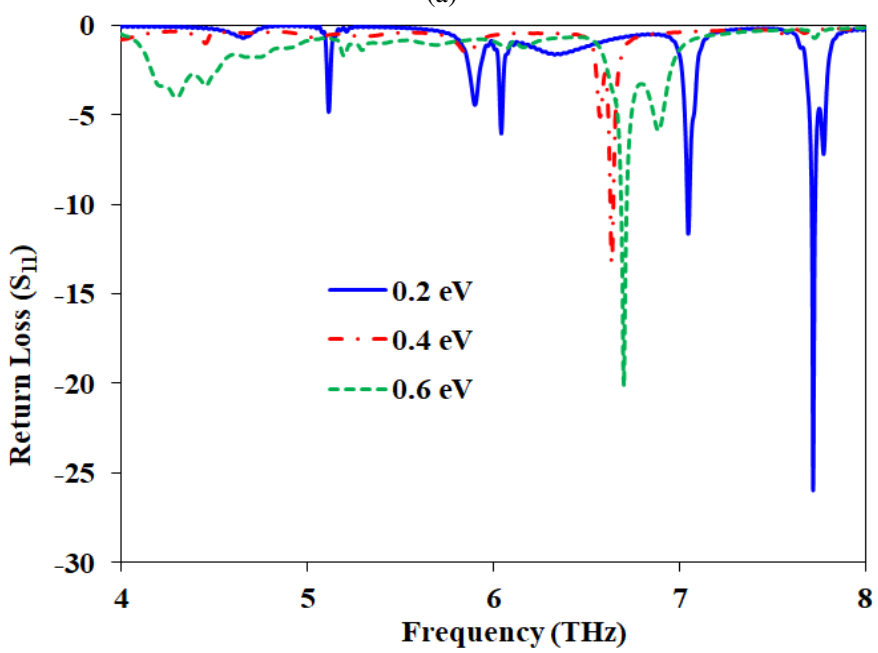

(b)

Fig.3 the reflection of the absorber with chemical potential of $0.2 \mathrm{eV}$ for graphene ribbon and various chemical potential of the main element (a) for primary absorber (b) for final absorber

To understand the effect of graphene ribbons, we assumed the disk potential to be $0.6 \mathrm{eV}$ and changed the chemical potential of the graphene ribbons e to $0.2,0.4$, and $0.6 \mathrm{eV}$, and the results are shown in Fig.4 (a). Here it is observed that with the increasing chemical potential of the graphene ribbons, the resonances are transferred to a higher frequency of $7.5 \mathrm{THz}$, and the reflection is increased to -10 $\mathrm{dB}$, so the chemical potential of the graphene ribbons is reduced to $0.2 \mathrm{eV}$ gives the best reflection. When we assume that the chemical potential of the ring and the disk is constant and the chemical potential of the graphene ribbons changes, we observe behavior in Fig.4 (b) that we saw earlier in Fig. 4 (a) for the structure of a disk without a ring, except that the ring reduces the reflection from the absorber. In fact, the loop prevents the scattering of fields and thus creates a capacitor around the disk, causing energy storage as a result of increasing the $\mathrm{Q}$ factor of the structure.

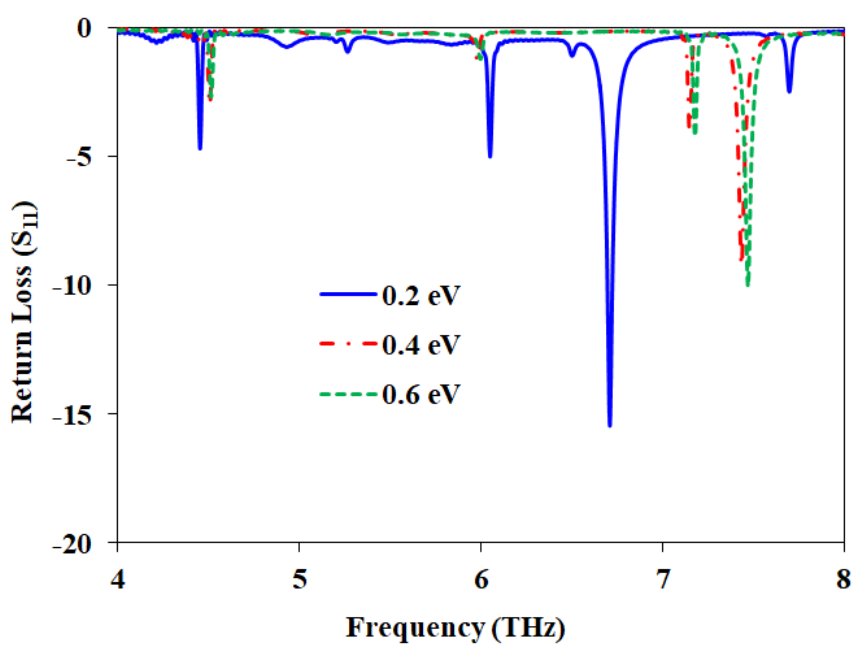

(a)

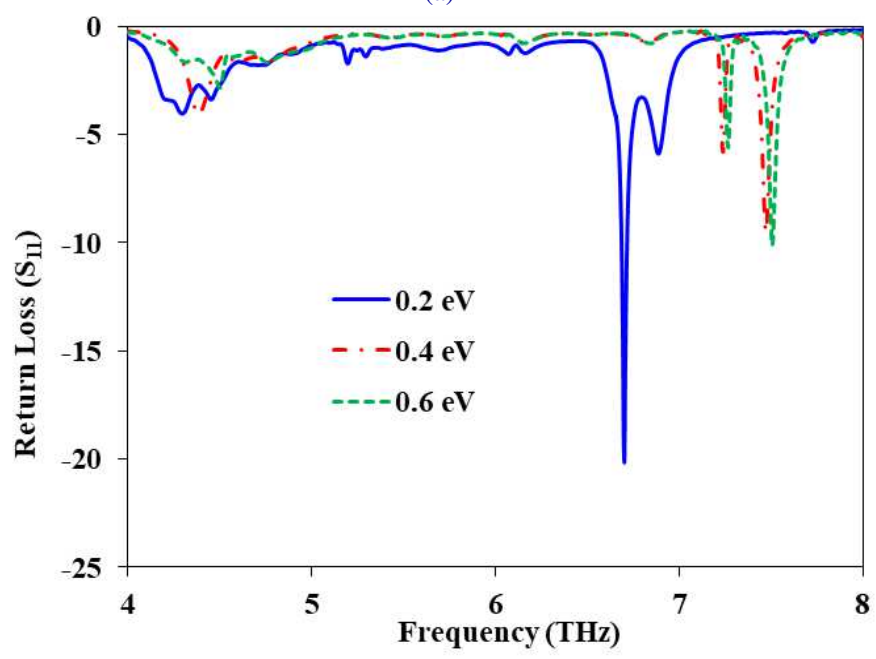

(b)

Fig.4 the reflection of the absorber with chemical potential of $0.6 \mathrm{eV}$ for the main element and various chemical potential of the graphene ribbon (a) for primary absorber (b) for final absorber

As shown in Fig.5, we examine the effect of relaxation time on the reflection of the absorber. We checked the relaxation time of graphene for the disk and ring in the final structure for the values of $0.5,1$ and $1.5 \mathrm{pS}$. As shown in the results, the relaxation time changes have no effect on the operating frequency, while the reflection value changes drastically, which also affects the Q-factor. The Q-factor of the proposed absorber for the relaxation time of $0.5 \mathrm{pS}$ is 239 but this amount is 670 and 1970 for relaxation time of $1 \mathrm{pS}$ and $1.5 \mathrm{pS}$ respectively. So, increasing the relaxation time can improve the Q-factor of the absorber. 


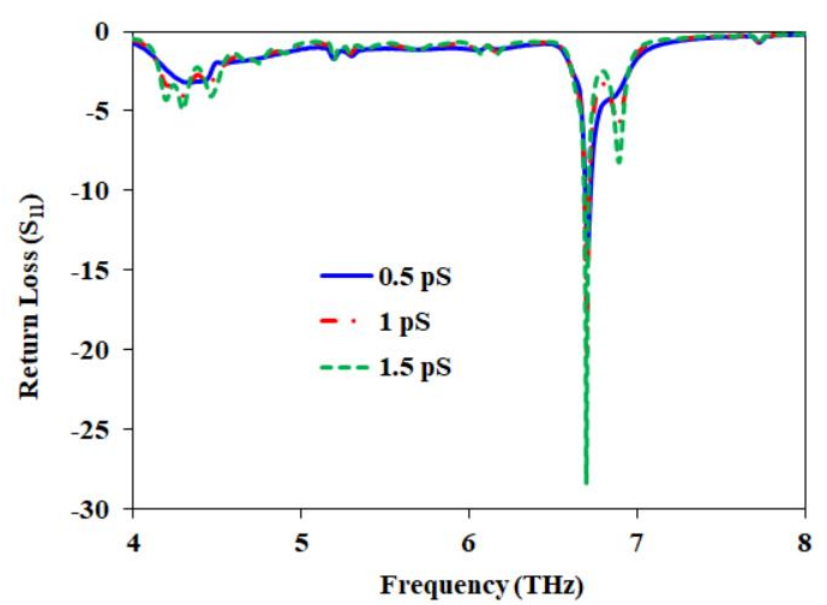

Fig.5 the effect of relaxation time for proposed structure on reflection

In Fig.6, the distribution of electric and magnetic fields for the structure of the disk at a frequency of $6.15 \mathrm{THz}$ at the absorber surface and the interface of the graphene ribbons and substrate is investigated. Fig. 6 (a) shows the value of electric field near graphene ribbons, which is about $136 \mathrm{~dB}$, which is the maximum field in the $\mathrm{X}$ direction, and Fig. 6 (b) shows the value of electric field at the absorber surface. It shows that it is about $148 \mathrm{~dB}$, and here the maximum field is clearly distributed in the $\mathrm{X}$ direction with dipole form. Fig. 6 (c) shows the magnitude of the magnetic field near graphene ribbons, which is about $90.7 \mathrm{~dB}$. In Fig. 6 (d) shows the amount of magnetic field at the absorber surface, which is about $95 \mathrm{~dB}$, and here the maximum field is clearly distributed inside the disk and actually creates an inductance property.

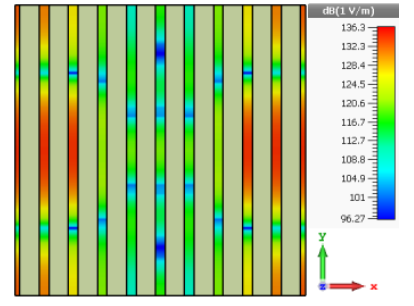

(a)

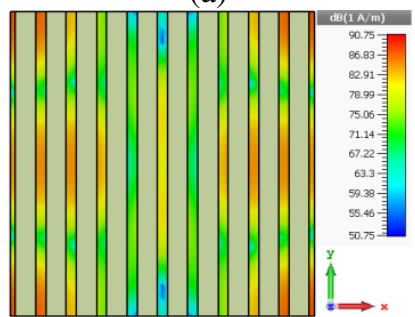

(c)

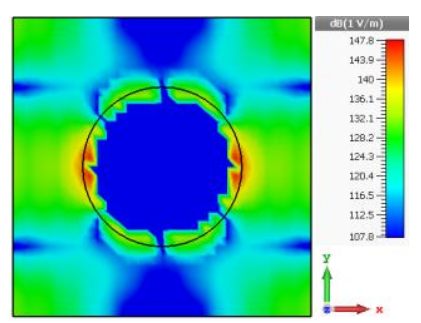

(b)

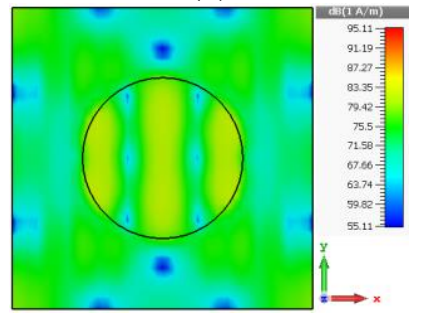

(d)
Fig.6 Field distribution for primary structure (a) Electric field on the graphene ribbons (b) Electric field in the absorber surface (c) Magnetic field on the graphene ribbons (d) Magnetic field in the absorber surface

In Fig.7, the distribution of electric and magnetic fields for the ring structure (final absorber) at a frequency of $6.70 \mathrm{THz}$ at the absorber surface and the interface of the graphene ribbons and substrate is investigated. Fig.7 (a) shows the electric field on the graphene ribbons, which is about $122 \mathrm{~dB}$. Fig. 7 (b) (b) shows the electric field at the absorber surface, which is about $139 \mathrm{~dB}$, and here the maximum field is clearly distributed in the $\mathrm{X}$ direction and dipole form. Fig. 7 (c) shows the magnitude of the magnetic field on the graphene ribbons, which is about $87.8 \mathrm{~dB}$ and Fig. 7 (d) shows the magnitude of the magnetic field at the absorber surface, which is about 85 $\mathrm{dB}$.

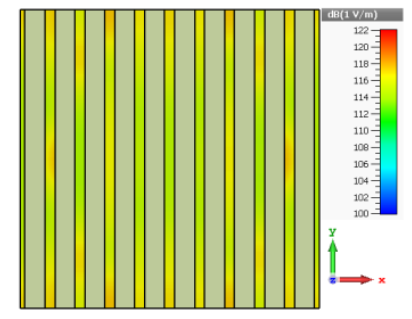

(a)

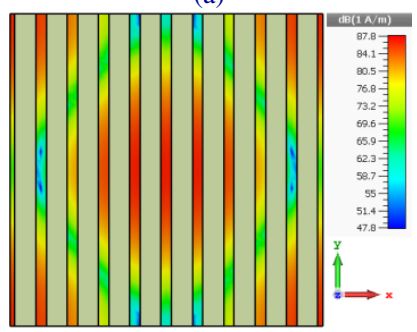

(c)

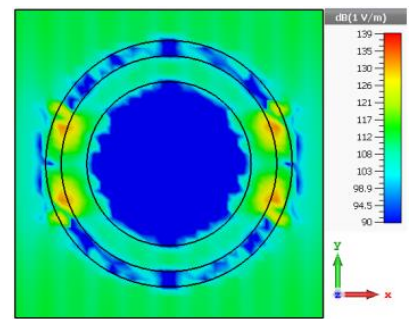

(b)

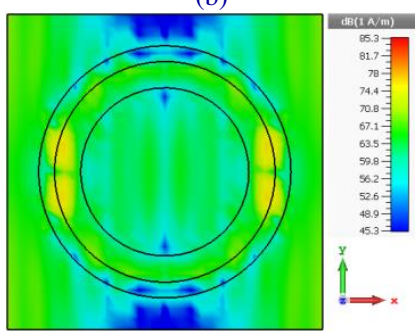

(d)
Fig.7 Field distribution for proposed structure (a) Electric field on the graphene ribbons (b) Electric field in the absorber surface (c) Magnetic field on the graphene ribbons (d) Magnetic field in the absorber surface

\section{Cancer detection with $\mathrm{THz}$ absorber}

As shown in Eq. 5, the Debye second order can be used to model breast and skin cancer and tissue [30]:

$\varepsilon(\omega)=\varepsilon_{\infty}+\frac{\varepsilon_{s}-\varepsilon_{2}}{1+i \omega \tau_{1}}+\frac{\varepsilon_{2}-\varepsilon_{\infty}}{1+i \omega \tau_{2}}$

The $\varepsilon_{\infty}, \varepsilon_{S}, \varepsilon_{2}, \tau_{1}$ and $\tau_{2}$ are temperature dependent of high frequency permittivity, static dielectric constant, intermediate frequency limit, slow relaxation time, and fast relaxation time. The parameters for breast cancer and breath healthy tissue are given in Table.1.

Table.1 Debye coefficients for healthy and cancerous breast tissue

\begin{tabular}{lccccc}
\hline & $\varepsilon_{\infty}$ & $\varepsilon_{S}$ & $\varepsilon_{2}$ & $\tau_{1}[\mathrm{Ps}]$ & $\tau_{2}[\mathrm{Ps}]$ \\
\hline Healthy tissue & 2.1 & 76.5 & 3.9 & 10.3 & 0.07 \\
Cancer tissue & 2.5 & 77.9 & 4.3 & 9.1 & 0.08 \\
\hline
\end{tabular}

In Fig. 8, the variation of the real and imaginary parts of the permittivity for healthy and cancerous breast tissue based on the second-order Debye model in the range of 4 to $8 \mathrm{THz}$. As can be seen, the cancerous tissue has a higher permittivity in the real and imaginary part, so we expect more frequency shifts and losses in the results for the cancerous tissue. 


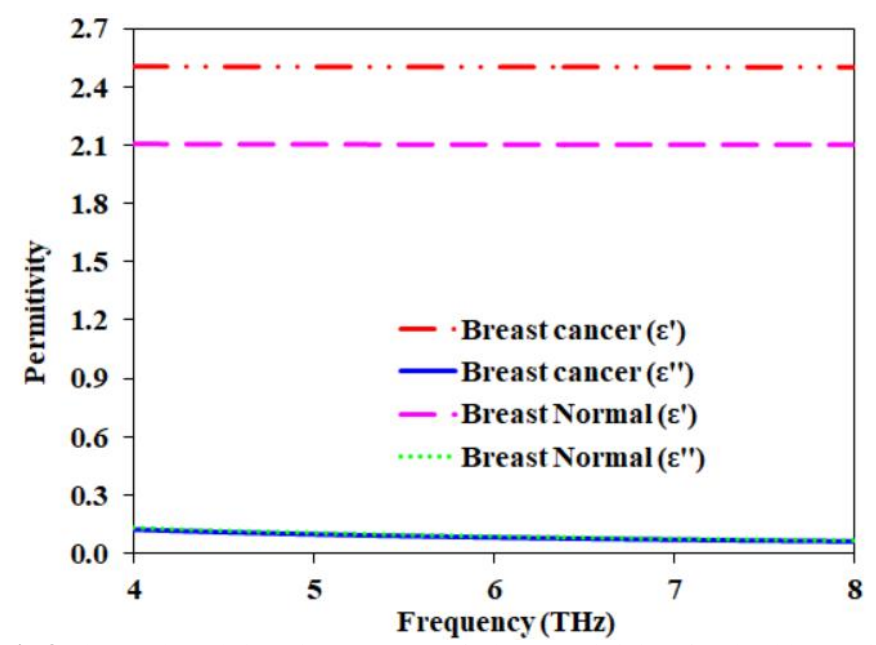

Fig.8 the real and imaginary parts of the permittivity for healthy and cancerous breast tissue

Here, we have considered two factors for cancer diagnosis and compared this issue for healthy and cancerous tissue. For breast cancer tissue, the results are presented in Fig. 9. Here we assume the distance of the object from the absorber is 0,5 and $10 \mu \mathrm{m}$, respectively. As can be seen in the figure, the frequency shift will be more by increasing distance for a constant thickness of $1 \mu \mathrm{m}$ of the sample tissue. When the tissue is placed on the absorber surface, the operating frequency is about $6.4 \mathrm{THz}$, but with increasing height, the working frequency has reached $5.8 \mathrm{THz}$. The reason for this phenomenon is the appearing a capacitance between the sample tissue and the absorber, which reduces the operating frequency. The sensitivity and FOM of the sensor can be used for determining the healthy and cancer cell when the tissues are placed in front of them. The sensitivity can be obtained by Eq.6 where the $\Delta f$ is the frequency shift and the variation of the refractive index is indicated by $\Delta n$ [30-31].

$S=\frac{\Delta f}{\Delta n}$

And the FOM can be calculated by Eq. (7), where the $S$ is the sensitivity of the sensor and FWHM (Full width at half maximum) is the $3 \mathrm{~dB}$ bandwidth of the reflection [30-31].

$F O M=\frac{S}{F W H M}$

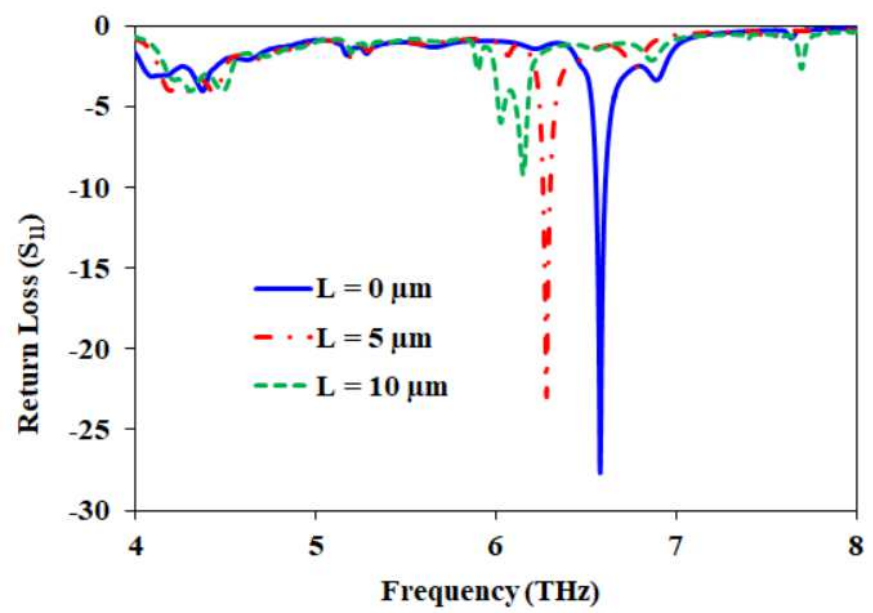

(a)

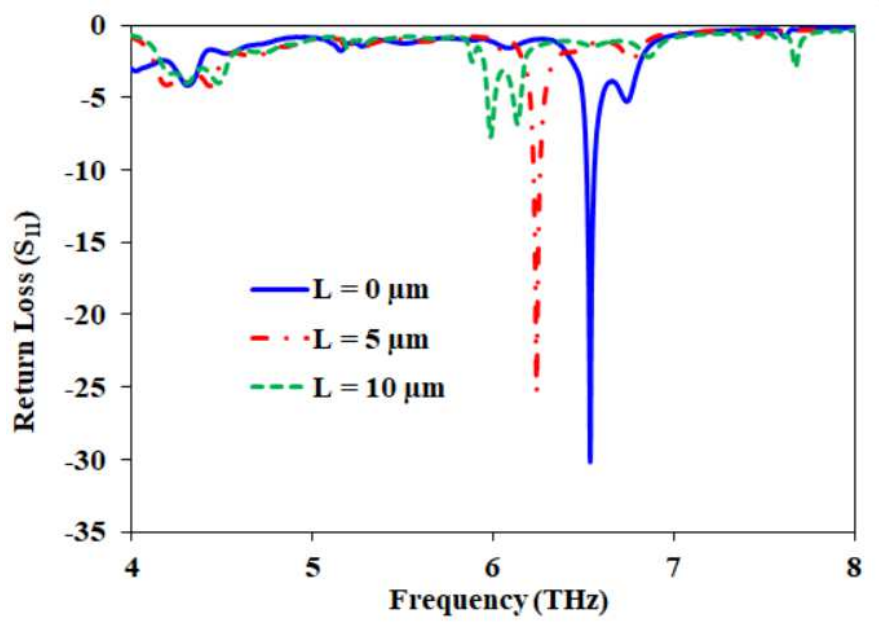

(b)

Fig.9 Effect of tissue distance from absorber (a) Healthy tissue for breast tissue (b) Cancer tissue for breast tissue

In Fig. 10, the effect of tissue thickness change on the frequency response is examined. The healthy and cancerous breast tissues with thicknesses of 1,3 and $5 \mu \mathrm{m}$ are placed on the absorber surface. Obviously, by increasing thickness, the capacitance will increase and as a result, the frequency shift will increase. Also, the frequency shift is higher for cancerous tissue than for healthy tissue.

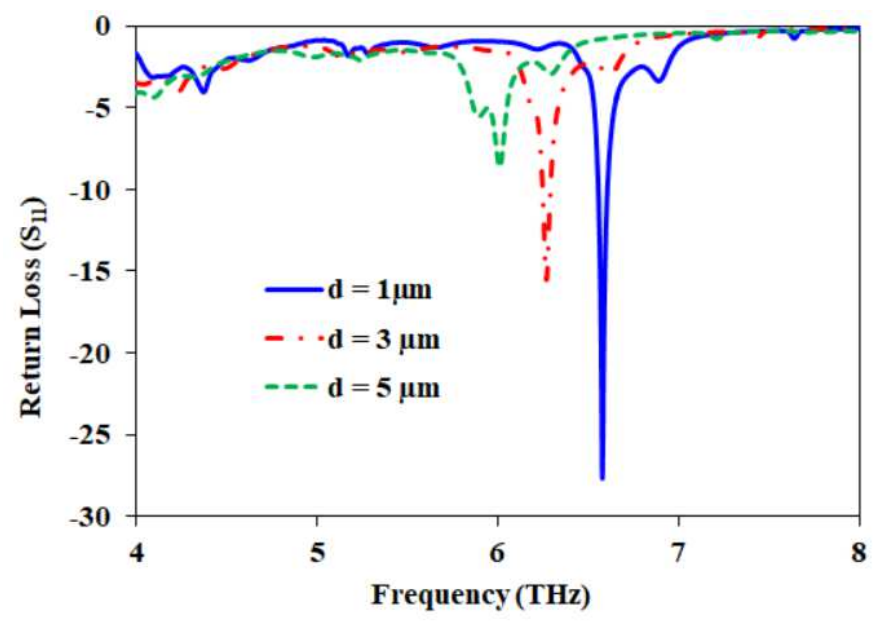

(a) 


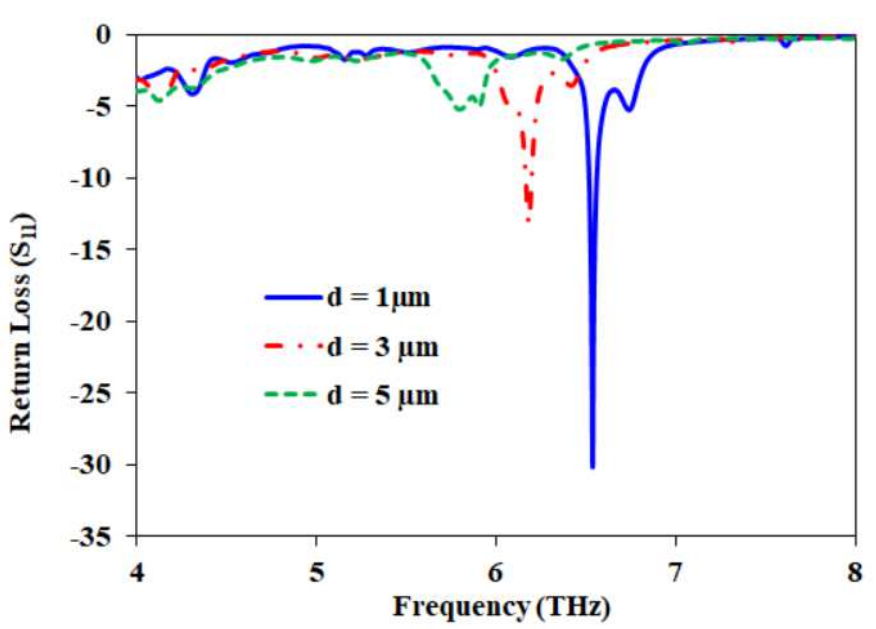

(b)

Fig.10 Effect of tissue thickness on absorber (a) Healthy tissue for breast tissue (b) Cancer tissue for breast tissue

The result for sensitivity and FOM for each case of our studied based on Eq. 6 and 7 are obtained and the presented in Table. 2 for various distance. Then, the effect of thickness on sensitivity and FOM are presented in Table.3. As shown in Table .2 and 3, the FOM and sensitivity reveal the discrimination between the healthy and cancer tissue clearly and we can use it for detecting the cancer very fast and accurate in the $\mathrm{THz}$ spectrum with a small sample. The sensitivity of the sensor will increase by increasing the thickness and distance of the tissues as shown inTable.2 and 3.

Table.2 the sensitivity and FOM of the sensor for healthy and cancer tissue for various distance

\begin{tabular}{ccccc}
\hline & & $\mathrm{L}=0 \mu \mathrm{m}$ & $\mathrm{L}=5 \mu \mathrm{m}$ & $\mathrm{L}=10 \mu \mathrm{m}$ \\
\hline $\begin{array}{c}\text { Sensitivity } \\
\text { (GHz/RIU) }\end{array}$ & Healthy tissue & 82 & 289 & 379 \\
& Cancer tissue & 101 & 292 & 458 \\
FOM & Healthy tissue & 10344 & 24137 & 9482 \\
& Cancer tissue & 29117 & 18312 & 7643 \\
\hline
\end{tabular}

Table. 3 the sensitivity and FOM of the sensor for healthy and cancer tissue for various thickness

\begin{tabular}{ccccc}
\hline & & $\mathrm{d}=1 \mu \mathrm{m}$ & $\mathrm{d}=3 \mu \mathrm{m}$ & $\mathrm{d}=5 \boldsymbol{\mu m}$ \\
\hline \multirow{2}{*}{ Sensitivity } & Healthy tissue & 82 & 296 & 475 \\
& Cancer tissue & 10344 & 9267 & 5172 \\
\multirow{2}{*}{ FOM } & Healthy tissue & 101 & 335 & 590 \\
& Cancer tissue & 29117 & 10470 & 1605 \\
\hline
\end{tabular}

\section{Conclusion}

In this paper, a narrowband $\mathrm{THz}$ absorber was developed for cancer detection. As the first point, the effect of the layer to improve the Q-factor of the absorber was shown and the ring element plays important rule for trapping the electric field in a limited area for the proposed absorber. The results show the effect of thickness and distance of the sample from absorber on sensitivity and FOM which can be considered to discriminate the cancer and healthy tissue.

\section{References:}

[1] Samsuzzaman, Md, Mohammad T. Islam, Md T. Islam, Abdullah AS Shovon, Rashed I. Faruque, and Norbahiah Misran. "A 16-modified antipodal Vivaldi antenna array for microwave-based breast tumor imaging applications." Microwave and Optical Technology Letters 61, no. 9 (2019): 2110-2118.

[2] Poorgholam-Khanjari, Shima, and Ferdows B. Zarrabi. "Reconfigurable Vivaldi $\mathrm{THz}$ antenna based on graphene load as hyperbolic metamaterial for skin cancer spectroscopy." Optics Communications 480 (2021): 126482.

[3] Balaji, Aditya, and Jin Zhang. "Electrochemical and optical biosensors for early-stage cancer diagnosis by using graphene and graphene oxide." Cancer nanotechnology 8, no. 1 (2017): 1-12.

[4] Shao, Wenyi, and Ryan S. Adams. "Two antipodal vivaldi antennas and an antenna array for microwave early breast cancer detection." Microwave and optical technology letters 55, no. 3 (2013): 670-674.

[5] Ruvio, Giuseppe, Raffaele Solimene, Antonietta D'Alterio, Max J. Ammann, and Rocco Pierri. "RF breast cancer detection employing a noncharacterized vivaldi antenna and a MUSIC-inspired algorithm." International Journal of $R F$ and Microwave ComputerAided Engineering 23, no. 5 (2013): 598-609.

[6] Rahman, Anis, Aunik K. Rahman, and Babar Rao. "Early detection of skin cancer via terahertz spectral profiling and 3D imaging." Biosensors and Bioelectronics 82 (2016): 64-70.

[7] Yu, Liu, Liu Hao, Tang Meiqiong, Huang Jiaoqi, Liu Wei, Dong Jinying, Chen Xueping, Fu Weiling, and Zhang Yang. "The medical application of terahertz technology in non-invasive detection of cells and tissues: opportunities and challenges." RSC advances 9, no. 17 (2019): 9354-9363.

[8] Woodward, Ruth M., Bryan E. Cole, Vincent P. Wallace, Richard J. Pye, Donald D. Arnone, Edmund H. Linfield, and Michael Pepper. "Terahertz pulse imaging in reflection geometry of human skin cancer and skin tissue." Physics in Medicine \& Biology 47, no. 21 (2002): 3853.

[9] Kazemi, Fatemeh. "High Q-factor compact and reconfigurable $\mathrm{THz}$ aperture antenna based on graphene loads for detecting breast cancer cells." Superlattices and Microstructures 153 (2021): 106865.

[10] Keshavarz, Afsaneh, and Zohreh Vafapour. "Water-based terahertz metamaterial for skin cancer detection application." IEEE Sensors Journal 19, no. 4 (2018): 1519-1524.

[11] Sadeghzadeh, Ramezan Ali, and Ferdows B. Zarrabi. "Metamaterial Fabry-Perot cavity implementation for gain and bandwidth enhancement of THz dipole antenna." Optik 127, no. 13 (2016): 5181-5185.

[12] Azizi, Shabnam, Shohreh Nouri-Novin, Mir Mohsen Seyedsharbaty, and Ferdows B. Zarrabi. "Early skin cancer detection sensor based on photonic band gap and graphene load at terahertz regime." Optical and Quantum Electronics 50, no. 6 (2018): 1-14.

[13] Nourinovin, Shohreh, and Akram Alomainy. "A Terahertz Electromagnetically Induced Transparency-Like Metamaterial for Biosensing." In 2021 15th European Conference on Antennas and Propagation (EuCAP), pp. 1-5. IEEE, 2021.

[14] Landy, N. I, S. Sajuyigbe, J. J. Mock, D. R. Smith, and W. J. Padilla. "Perfect metamaterial absorber." Physical review letters 100, no. 20 (2008): 207402. 
[15] Fu, Yanjun, Shihao Li, Yan Chen, Xiaofan Zhang, and Kejian Chen. "A multi-band absorber based on a dual-trident structure for sensing application." Optical and Quantum Electronics 53, no. 2 (2021): 1-11.

[16] Wen, Qi-Ye, Huai-Wu Zhang, Yun-Song Xie, Qing-Hui Yang, and Ying-Li Liu. "Dual band terahertz metamaterial absorber: Design, fabrication, and characterization." Applied Physics Letters 95, no. 24 (2009): 241111.

[17] Arezoomand, Afsaneh Saee, Ferdows B. Zarrabi, Samaneh Heydari, and Navid P. Gandji. "Independent polarization and multi-band $\mathrm{THz}$ absorber base on Jerusalem cross." Optics Communications 352 (2015): 121-126.

[18] Tavakoli, Fatemeh, Ferdows B. Zarrabi, and Hamed Saghaei. "Modeling and analysis of high-sensitivity refractive index sensors based on plasmonic absorbers with Fano response in the near-infrared spectral region." Applied optics 58, no. 20 (2019): 5404-5414.

[19] Soheilifar, Mohammad Reza. "Wideband optical absorber based on plasmonic metamaterial cross structure." Optical and Quantum Electronics 50, no. 12 (2018): 1-12

[20] Mobasser, Shohreh, Shima Poorgholam-Khanjari, Maryam Bazgir, and Ferdows B. Zarrabi. "Highly Sensitive Reconfigurable Plasmonic Metasurface with Dual-Band Response for Optical Sensing and Switching in the Mid-Infrared Spectrum." Journal of Electronic Materials 50, no. 1 (2021): 120-128.

[21] Fu, Pan, Fei Liu, Guang Jun Ren, Fei Su, Dong Li, and Jian Quan Yao. "A broadband metamaterial absorber based on multi-layer graphene in the terahertz region." Optics Communications 417 (2018): 62-66

[22] Peng, Lin, Xiao-ming Li, Xiao Liu, Xing Jiang, and Si-min Li. "Metal and graphene hybrid metasurface designed ultra-wideband terahertz absorbers with polarization and incident angle insensitivity." Nanoscale Advances 1, no. 4 (2019): 1452-1459.

[23] Cen, Chunlian, Jiajia Chen, Cuiping Liang, Jing Huang, Xifang Chen, Yongjian Tang, Zao Yi, Xibin Xu, Yougen Yi, and Shuyuan Xiao. "Plasmonic absorption characteristics based on dumbbell-shaped graphene metamaterial arrays." Physica E: Low-dimensional Systems and Nanostructures 103 (2018): 93-98.

[24] Li, Hongju, Lingling Wang, and Xiang Zhai. "Tunable graphene-based mid-infrared plasmonic wide-angle narrowband perfect absorber." Scientific reports 6, no. 1 (2016): 1-8.

[25] Biabanifard, Mohammad, Somayyeh Asgari, Sadegh Biabanifard, and Mohammad Sadegh Abrishamian. "Analytical design of tunable multiband terahertz absorber composed of graphene disks." Optik 182 (2019): 433-442.

[26] Zhou, Qihui, Peiguo Liu, Li-an Bian, Hanqing Liu, Chenxi Liu, and Genghui Chen. "Controlling enhanced absorption in graphene metamaterial." Optics Communications 413 (2018): 310-316.

[27] Zarrabi, Ferdows B., Mojtaba Mohaghegh, Maryam Bazgir, and Afsaneh Saee Arezoomand. "Graphene-Gold Nano-ring antenna for Dualresonance optical application." Optical Materials 51 (2016): 98-103.

[28] Soheilifar, Mohammad Reza, and Ferdows B. Zarrabi. "Reconfigurable metamaterial absorber as an optical switch based on organic-graphene control." Optical and Quantum Electronics 51, no. 5 (2019): 1-12.

[29] Ebrahimi, Sepideh, and Shima Poorgholam-Khanjari. "Highly Q-factor optical metasurface based on DNA nanorods with Fano response for onchip optical sensing." Optik 232 (2021): 166576.

[30] Vafapour, Zohreh, Afsaneh Keshavarz, and Hossain Ghahraloud. "The potential of terahertz sensing for cancer diagnosis." Heliyon 6, no. 12 (2020): e05623.

[31] Poorgholam-Khanjari, Shima, Zahra Razavi, and Ferdows B. Zarrabi. "Reconfigurable optical rectangular particle array absorber based on metal-DNA-metal structure as a refractive index sensor and optical switch." Optics Communications 489 (2021): 126866. 